\title{
Erfolge der nordischen Wirtschafts- und Sozialmodelle im europäischen Vergleich
}

\author{
Ognian N. Hishow*
}

Die kleinen nordischen Nationen Schwedens, Finnlands und Dänemarks gelten seit jeher als wohlhabend, friedlich und sozial fürsorglich. Der hohe Lebensstandard der Skandinavier war insbesondere nach dem Zweiten Weltkrieg in Europa sprichwörtlich, da militärische Auseinandersetzungen im 20. Jahrhundert keine nennenswerten ökonomischen Schocks verursacht haben. Das Pro-Kopf-Einkommen Schwedens und Dänemarks war noch Mitte der 1960er Jahre das höchste unter den 15 Ländern der alten Europäischen Union, und die Sozialleistungen des Staates galten quantitativ und qualitativ als weltweit einmalig. Zentral für das skandinavische Wirtschafts- und Sozialmodell ist der breite gesellschaftliche Konsens über die Notwendigkeit und Aufrechterhaltung eines umfassenden Wohlfahrtsstaates. Eine Direktübertragung des nordischen Systems auf Deutschland und die anderen großen europäischen Volkswirtschaften ist aus strukturellen und historischen Gründen nicht möglich; einzelne wirtschaftspolitische Elemente können jedoch übernommen werden.

Ein weiteres Charakteristikum war in der Vergangenheit das relativ geringe Wirtschaftswachstum. Während das Nachkriegswachstum in Kontinentaleuropa (ohne den Ostblock) bis in die 1970er Jahre hinein noch dynamisch war, verzeichneten Schweden und Dänemark geringere jährliche Wachstumsraten. Das war angesichts der hohen Pro-Kopf-Einkommen jedoch kein weiteres Problem. Finnland, das um 1960 rund 40 Prozent ärmer als die anderen beiden nordischen Länder war, erzielte kontinuierlich höhere Wachstumsraten seiner Volkswirtschaft und konnte um 1975 aufschließen.

Die langfristig geringere Wirtschaftsdynamik Schwedens und Dänemarks beziehungsweise die Wachstumsverlangsamung in Finnland nach dem Zerfall der ehemaligen Sowjetunion bewirkten, dass die nordischen Mitglieder der Europäischen Union gegenwärtig nicht mehr unions- und OECD-weit im Pro-Kopf-Einkommen führend sind (Abb. 1).

\section{Abbildung 1: Pro-Kopf-Einkommen zu Kaufkraftparitäten relativ zum Unions- durchschnitt (EU-15=100 Prozent)}

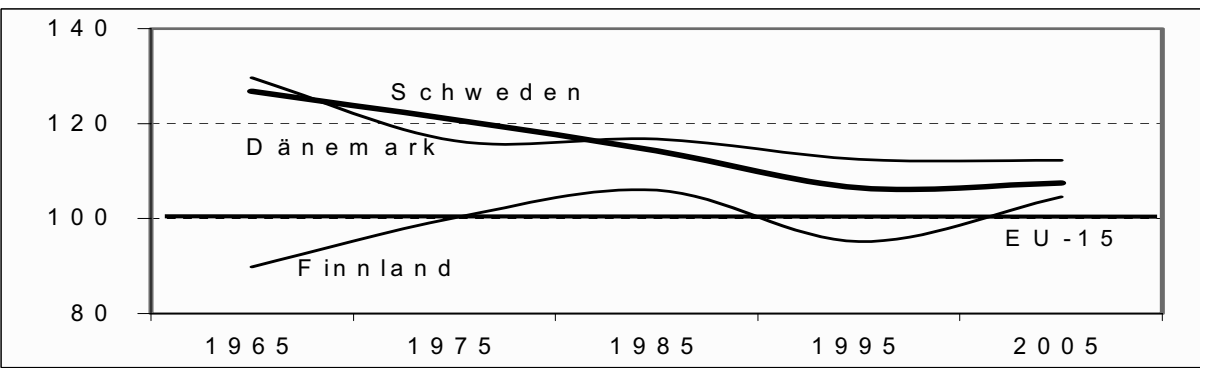

Quelle: European Commission, DG EcFin, European Economy, No. 6/2004, Table 9

* Dr. Ognian N. Hishow, Stiftung Wissenschaft und Politik, Berlin. 
Sie haben sich in der Nachkriegszeit dem Durchschnitt der 15 westlichen Mitglieder angenähert, wobei der relative Positionsverlust sogar noch deutlicher wäre, wenn nicht die gewichtigste europäische Volkswirtschaft - Deutschland - ebenfalls eine spürbare relative Verschlechterung verzeichnet hätte. Was jedoch die skandinavischen Gesellschaften vom Rest der Union unterscheidet, ist der hohe Grad an sozialer Kohäsion, ausgedrückt in den OECD-weit niedrigsten Einkommensunterschieden nach Steuern. Der kleine Gini-Koeffizient insbesondere in Dänemark und Schweden von weniger als 0,25 (Finnland liegt mit 0,25 leicht darüber ${ }^{1}$ ) erklärt sich aus der Kombination aus Besteuerung und Transfers, die in diesen Ländern intensiver sind als im Durchschnitt der Union bzw. der OECD.

Ferner sind die drei Länder besser als die meisten anderen Partner mit den jüngsten Herausforderungen der Osterweiterung und Globalisierung fertig geworden. Seit Mitte der 1990er Jahre verzeichnen Schweden und Dänemark ein höheres, Finnland ein deutlich höheres Wachstum als der Rest der alten Mitglieder, so dass der Trend des relativen Positionsverlustes zum Stillstand gekommen ist (Abb. 1). In allen drei Ländern konnten die Arbeitslosenraten reduziert werden. Mit Ausnahme Finnlands liegen sie derzeit unter dem Unionsdurchschnitt, wobei die sehr hohe finnische Arbeitslosigkeit in den letzten 10 Jahren halbiert werden konnte.

Im Zuge der Reformdebatte in der EU wird oft auf die wirtschaftspolitische Leistung der nordischen Mitglieder hingewiesen, die trotz - oder wegen - der hohen sozialen Standards erreicht wird. Vor dem Hintergrund der Reformdebatte in Deutschland und den anderen großen Kontinentalökonomien mit dringendem Reformbedarf - Frankreich und Italien - fällt auf, dass sich insbesondere die Ökonomen schwer tun, ein einheitliches Erklärungsmuster für das Phänomen ,nordischer Wirtschaftserfolg ' zu finden. Einerseits ist oft die Neigung unverkennbar, gerade die distributive Rolle des Staates für das gute Abschneiden der Skandinavier beim Wachstum und Haushaltssaldo zu identifizieren. ${ }^{2}$ Zur wirtschaftstheoretischen Begründung wird aus der Literatur das sogenannte Haavelmo-Theorem angeführt, wonach die steuerfinanzierte Staatsnachfrage voll wachstumswirksam wird. Die dahinter steckende Argumentation ist, dass die (aus dem Steueraufkommen getätigten) Käufe und Transfers des Staates nicht gespart und folglich nicht gemindert werden, so dass die gesamtwirtschaftliche Nachfrage und damit das Wachstum proportional zulegt.

Auf der anderen Seite wird erkannt, dass sich - abgesehen von der Kritik am theoretischen Ansat $^{3}$ - die als positiv wahrgenommene skandinavische Erfahrung mit einer massiven BIP-Umverteilung über den Staat nicht einfach auf andere Volkswirtschaften übertragen lässt. Diesbezügliche Beispiele für Volkswirtschaften mit einer großen Staatsquote, die an ihre Wachstums-, Beschäftigungs- und fiskalischen Grenzen stoßen, liefern zahlreiche früher erfolgreiche westeuropäische Länder - neben Deutschland insbesondere Belgien, Frankreich und Italien. Die Massenunruhen im Herbst 2005 in französischen Vorstädten zeigten erneut, dass hohe Staatsquoten und selbst umfangreiche Sozialtransfers allein nicht ausreichen, um den sozialen Zusammenhalt der Gesellschaft zu sichern, wenn keine Beschäfti-

1 Der (in der Regel standardisierte) Gini-Koeffizient der Verteilung des Volkseinkommens nimmt Werte zwischen Null und Eins an. Im ersteren Fall würden alle Haushalte das gleiche Nettoeinkommen erzielen; im letzteren würde das gesamte Volkseinkommen auf einen Haushalt entfallen. Angaben für 2000. Quelle: OECD, Economic Survey Denmark, Finland, Sweden, Paris 2004, 2005.

2 Vgl. Paul de Grauwe/Magdalena Polan: Globalisation and social spending, CesIfo working paper No. 885, München, März 2003; Karl Aiginger: Towards a new European model of a reformed welfare state: An alternative to the United States model, in: UN ECE, Economic Survey of Europe, 1/2005, Genf 2005, Kapitel 7.

3 Ein wesentlicher Kritikpunkt ist, dass bei steigender Steuerquote der Einkommensmultiplikator kleiner wird. Im Extremfall - bei sehr hoher Besteuerung - kann er unwirksam werden, so dass die zusätzliche Staatsnachfrage kein Wachstum mehr generiert. 
gungschancen bestehen. Im Gegenteil, sie geben wohl der traditionellen angelsächsischen Einstellung Recht, wonach sozial alles ist, was Jobs schafft.

Ferner ist der Homogenitätsgrad des nordischen Modells geringer als allgemein perzipiert. Die Gemeinsamkeiten sind auf der Nachfrageseite größer; insbesondere fallen die redistributiven Effekte der Besteuerung und der Transfers hier stärker ins Gewicht, als in den meisten anderen europäischen und OECD-Länden. Auf der Angebotsseite sind bei den skandinavischen Mitgliedern der Europäischen Union weniger gemeinsame Beschäftigungs- und steuerpolitische Grundsätze auszumachen; vielmehr gehen die nordischen Länder oft verschiedene wirtschaftspolitische Wege.

\section{Beschäftigung und Arbeitskosten}

Die unterschiedliche Beschäftigungssituation ist mit die gravierendste Differenz zwischen den skandinavischen Mitgliedern der Europäischen Union. In den 1990er Jahren musste die finnische Volkswirtschaft eine integrationspolitische Wende vollziehen - weg von der Verflechtung mit den kollabierenden Wirtschaften Russlands und der GUS und hin zur EU. Wegen der höheren Anforderungen der westlichen Märkte an die Qualitäts- und Technologiestandards fand auch ein Wandel innerhalb der finnischen Erzeugnisstruktur statt, der den Anteil der Medium-Tech- zugunsten der High-Tech-Erzeugnisse im finnischen Export verringerte. Voraussetzung und Erfordernis dieser technologischen Wende war zwangsläufig eine spürbare Produktivitätserhöhung, die wegen der daraus folgenden Umstrukturierungen und Entlassungen die Arbeitslosenrate in die Höhe schnellen ließ. Auf dem Höhepunkt der Beschäftigungskrise im Jahre 1994 erreichte die Arbeitslosigkeit 16,6 Prozent und lag damit um mehrere Prozentpunkte über der deutschen, französischen oder italienischen Rate jener Zeit. $^{4}$

Dänemarks und Schwedens Volkswirtschaften mussten auf Grund ihrer traditionellen Verflechtung mit der EU zwar nicht einen gravierenden außenwirtschaftlichen Wandel vollziehen; sie mussten aber im Zuge der voranschreitenden Globalisierung dennoch Anpassungen vornehmen. Mit 9,6 Prozent und 9,9 Prozent erreichte die Arbeitslosigkeit in Dänemark und Schweden 1993 bzw. 1997 einen vorläufigen historischen Höhepunkt. ${ }^{5}$ Zum Vergleich: Die Arbeitslosenrate war im wiedervereinigten Deutschland damals noch geringer. Sowohl Dänemark als auch Schweden gingen im Grunde den auch in Deutschland viel diskutierten Weg der Umverteilung der Arbeit - sie kürzten etwa die geleisteten Arbeitsstunden je Beschäftigten bei gleichzeitiger leichter Steigerung der Erwerbsquote. Im Ergebnis werden in Schweden und Dänemark zur Zeit mehr Arbeitsstunden pro Jahr und Einwohner geleistet als in den langsam wachsenden europäischen Volkswirtschaften Frankreich, Italien und Deutschland. ${ }^{6}$

Auch in Finnland werden deutlich mehr Jahresarbeitsstunden pro Einwohner als beispielsweise in Deutschland geleistet. Nur sind die Arbeitsstunden anders als in den skandinavischen Nachbarländern verteilt: Sie sind je Beschäftigten auf einem höheren Stand als in Schweden und Dänemark geblieben. In Kombination mit einer geringeren Erwerbsquote als dort liefert dies eine Erklärung für die höhere finnische Arbeitslosenrate.

Eine Verteilung der Arbeit ,auf mehr Schulter“ setzt eine entsprechende Anpassung der Arbeitskosten voraus, damit die weniger produktiven Beschäftigten nicht vom Arbeitsmarkt verdrängt werden. Die Lohnquote - der Lohnanteil am BIP - in Schweden passte sich dem

4 EU Commission, DG EcFin, Reihe European Economy, 6/2004, S. 418.

5 EU Commission, DG EcFin, Reihe European Economy, 6/2004, S. 418.

6 OECD, Economic Survey France, 10/2005, Paris, S. 100. 
Konjunkturzyklus sowohl in den 1990ern als auch nach 2000 an - in der Phase konjunktureller Abschwächung 1991/92 und 2001/2002 ging sie zurück und wirkte somit antizyklisch.

Allerdings sind auch in anderen Ländern, beispielsweise in Deutschland und Frankreich, die Lohnstückkosten in den letzten Jahren zurückgegangen, in Deutschland jedoch von einem im internationalen Vergleich hohen Stand aus. Nur ist in diesen Ländern entweder das Wachstum langsamer (Deutschland), oder die Arbeitslosigkeit größer (in beiden). Dabei sind die Lohnnebenkosten (Arbeitnehmer - zuzüglich des Arbeitgeberanteils der Sozialversicherung) in Schweden und Deutschland etwa gleich hoch (während die französischen darüber liegen).

Eine Erklärung, warum das skandinavische Land seine Arbeitslosigkeit unter Kontrolle hat, liefert die internationale Kosten- und Produktivitätsstruktur: In Schweden sind die Bruttolöhne im Durchschnitt der Volkswirtschaft 20 Prozent geringer als in Deutschland. Zugleich liegt die schwedische Stundenproduktivität nur unwesentlich unterhalb der deutschen, woraus sich eine günstigere Wettbewerbsposition Schwedens ergibt, da die Lohnstückkosten in dem nordischen Land niedriger sind. ${ }^{7}$

Dagegen versagt dieses klassische Erklärungsmuster im Falle Frankreichs: Die französischen Lohnstückkosten sind deutlich, um rund 17 Prozent, niedriger als die schwedischen. Warum dies nicht eine größere Beschäftigung in Frankreich nach sich zieht ist auf Grund des Arbeitsinputs und der Qualifikationsstruktur zu verstehen. In Frankreich werden - gesetzlich bestimmt - weniger Arbeitsstunden pro Jahr als in Schweden geleistet, was die Wirtschaft dazu zwingt, Sektoren und Branchen mit geringer Produktivität ,aufzugeben'. Würde man auf die brachliegende Reserve der Arbeitslosen in großem Stil zurückgreifen würde die Produktivität fallen und die Wettbewerbsfähigkeit (in Gestalt steigender Lohnstückkosten) der Unternehmen zurückgehen. Dagegen bringt die günstigere Arbeitszeitregelung in Schweden wirtschaftliche Vorteile und letztendlich ein größeres Beschäftigungspotential mit sich.

\section{Abbildung 2: Lohnnebenkosten 2003 in der EU/OECD (in Prozent des Bruttolohns)}

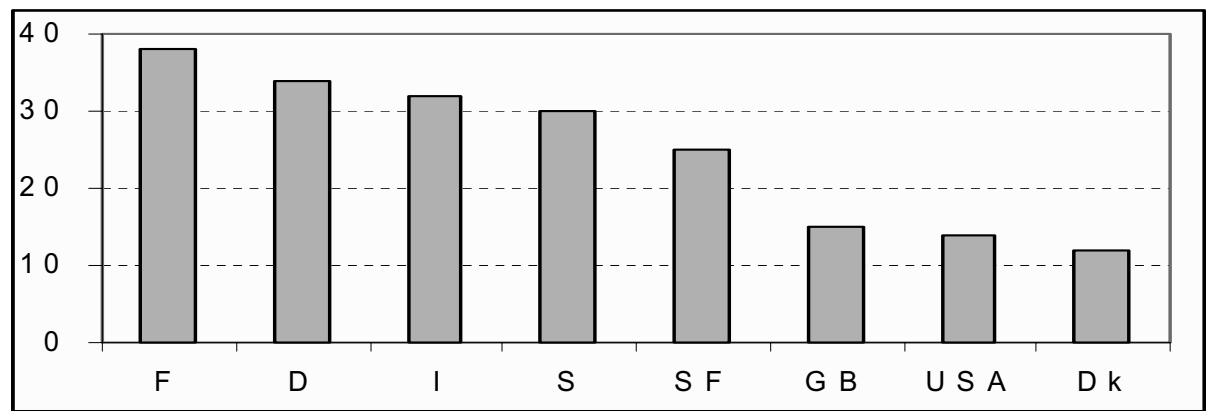

Quelle: OECD, Taxing Wages, http://www.oecd.org/dataoecd/26/8/2495227.pdf (letzter Zugriff: 20.12.2005)

Für Dänemark muss nach einem anderen Erklärungsmuster für die geringe Arbeitslosenrate im Vergleich zu Deutschland und Frankreich gesucht werden. Hier spielen die Lohnnebenkosten die Hauptrolle, die mit 12 Prozent des Bruttolohns die niedrigsten in der EU

7 Zu den Arbeitskosten: OECD, Taxing Wages: 2002-2003 - 2003 Edition, Tabelle I.3.; Zur Stundenproduktivität: OECD zit. nach Robert McGuckin/Bart Van Ark: New Member States Will Rise Produkctivity, in: The Conference Board, Executive Action, Nr. 89, 2004, Tabelle 4. 
sind. ${ }^{8}$ Mehr noch, der Arbeitgeberanteil ist praktisch Null. Im Ergebnis sind die Bruttolöhne in Dänemark geringer als in Deutschland und nur unwesentlich höher als in Finnland mit seinem doppelt so hohen Anteil der Lohnnebenkosten. Die Kombination aus etwas geringerem Bruttolohn und spürbar geringeren Lohnnebenkosten machen den Standort Dänemark selbst bei einer traditionellen Wirtschaftsstruktur ohne hohe High-Tech-Anteile attraktiv. Ursache ist, dass nach Abzug der Lohnnebenkosten vom ,Brutto', im Vergleich zu anderen Hochlohnländern mehr ,Netto “ übrig bleibt. ${ }^{9}$ Das wirkt mäßigend auf die Lohnentwicklung und mildert den Verdrängungseffekt in den Sektoren mit geringer Produktivität, die - wie das Beispiel der Länder mit hoher Arbeitslosigkeit zeigt - besonders unter dem Effekt der Lohnnebenkosten leiden.

Neben den Steuern und Abgaben ist die Regulierungsdichte ein wichtiger Standortfaktor, da Eingriffe in die Beschäftigungsstrategie der Unternehmen kostenwirksam sind. Der OECD zufolge haben die skandinavischen Volkswirtschaften in den 1990er Jahren die größten Fortschritte beim Abbau der staatlichen Regulierung in der EU gemacht. ${ }^{10}$ Das verhalf den Ländern, einschließlich Finnlands, dessen Beschäftigungskrise besonders gravierend war, die Arbeitslosigkeit während der letzten 10 Jahre zu reduzieren. ,Flexicurity ‘ symbolisiert die Bemühungen des Staates, eine Schlechterstellung des Einzelnen im Ergebnis der Flexibilisierung zu vermeiden, insbesondere durch Abrutschen in schlechter bezahlte Beschäftigung. In Dänemark wurde unter Finanzminister Moggens Lykketoft in den 1990er Jahren das Prinzip der aktiven Einbeziehung der Arbeitslosen in ihre eigene Jobsuche auf der Grundlage individueller Aktionspläne, bis hin zur Zwangsausbildung (für Jugendliche ohne Ausbildung) eingeführt. Durch Jobtraining, Weiterbildung und Qualifizierung soll ein Produktivitäts- und Einkommensverlust vermieden werden. Zugleich wurde im Zuge der Flexibilisierung der Kündigungsschutz gelockert und die Zumutbarkeit bei schlechter bezahlten Anstellungen erweitert. ${ }^{11}$ Um die Kritik, der dänische Staat habe sich dem ,Hire and fire" - Prinzip verschrieben, zu entschärfen, wurde im Gegenzug das sehr hohe Versorgungsniveau von rund 90 Prozent des letzten Nettolohns beibehalten. Schweden bezieht rund ein Viertel der arbeitslosen Personen in Jobtraining- und Qualifizierungsmaßnahmen ein - andernfalls wäre die gemeldete Arbeitslosenrate höher. In den drei Ländern hat die Verbreitung von Zeitverträgen zu einer steigenden Partizipation der Frauen und damit zu einer höheren Erwerbsquote in Skandinavien geführt, wobei im Rahmen der Lissabon-Strategie Schweden und Dänemark die höchsten Erwerbsquoten für Frauen in der EU erzielen. Hier zahlt sich das umfassende System der Kinderbetreuung aus, da dadurch mehr Frauen dem flexiblen Segment des Arbeitsmarktes zur Verfügung stehen.

Insbesondere in Schweden und Dänemark haben die Gewerkschaften eine Politik moderater Lohnforderungen praktiziert, die im Sinne der skandinavischen Präferenz für Konsenslösungen stärker auf die Schaffung neuer Arbeitsplätze konzentriert war als auf die Erhaltung von Privilegien für die Insider. Studien belegen zudem eine schwache Korrelation zwischen dem gewerkschaftlichen Organisationsgrad und der Arbeitslosenrate in den Ländern. ${ }^{12}$

8 Daten zu Dänemark aus: OECD, Taxing Wages: 2002-2003, Tabelle I.3.

9 Dieses ,Netto“ ist nicht mit dem verfügbaren Einkommen zu verwechseln, das nach Abzug der Lohnsteuer ermittelt wird. Die Lohnsteuersätze beeinträchtigen die Standortqualität aber weniger stark als die Lohnnebenkosten.

10 Ulrich van Stumm: Beschäftigungsgefälle in Europa: Ursachen und Konsequenzen. In: Rolf Caesar/Konrad Lammers (Hrsg.): Konvergenz und Divergenz in der EU - Empirische Befunde und wirtschaftspolitische Implikationen, Nomos, 2003, S. 48.

11 Wolfgang Biermann: Umbau des ,nordischen Wohlfahrtstaates“. Hintergrundpapier, Norwegisch-Deutsche Willy-Brandt-Stiftung, in: http://www.willy-brandt-stiftung.de/umbau.pdf (letzter Zugriff: 20.12.2005), S. 4.

12 Dean Baker/Andrew Glyn/David Howell/John Schmitt: Labor Market Institutions and Unemployment: A Critical Assessment of the Cross-Country Evidence, in: CEPA Working Paper 2002-17, November 2002, S. 10. 
Alles in allem ist die aktuelle Lage auf dem Arbeitsmarkt im Vergleich zur Mitte der 1990er Jahre besser. Zwar ist der Durchschnitt der Arbeitslosenrate in den drei skandinavischen Mitgliedsländern (einschließlich der versteckten schwedischen Arbeitslosigkeit) größer als in den angelsächsischen Ökonomien Großbritannien und Irland (sowie den USA). Zugleich sind aber in Nordeuropa die Lohnspreizung und der Gini-Koeffizient kleiner als beim angelsächsischen Modell.

\section{Wachstum trotz hoher Steuer- und Staatsquote}

Was erklärt das höhere Wachstum in Skandinavien im Vergleich zu Deutschland vor dem Hintergrund einer größeren Staatsquote? Warum sind die Haushaltssalden positiv und die Gesamtverschuldung der nordischen Länder geringer als in vielen anderen europäischen Volkswirtschaften (z. B. Frankreich)?

Oft von der breiten Öffentlichkeit unbemerkt, haben die skandinavischen Mitglieder der Europäischen Union ihr Image als Hochsteuerländer teilweise korrigiert. Gegenwärtig melden Dänemark und Finnland geringere Staatsquoten als beispielsweise Frankreich, und die finnische Steuerquote liegt ebenfalls unter der französischen (siehe Abb. 3 und Tab. 1, 3. Zeile). Die drei nordischen Länder haben in den letzten 15 Jahren viel stärker als die anderen Hochsteuerländer in der EU ihre Ausgaben verringert. Charakteristisch für alle drei sind mittlerweile weniger die hohen Staatsanteile am BIP, sondern die mutige Kürzung dieser Anteile. In Schweden, Finnland und Dänemark wurde die Staatsquote von ihrem Maximum in den 1990er Jahren um rund 24, 22 bzw. 17 Prozent reduziert; in Frankreich beträgt die entsprechende Kürzung lediglich 3 Prozent, und auch in den meisten anderen Mitgliedsländern mit hohen Staatsanteilen fallen die Kürzungen geringer aus. Im Ergebnis haben sich die Skandinavier der durchschnittlichen Staatsquote der 15 alten Mitgliedsländern klar angenähert.

\section{Abbildung 3: Staatsquote in den nordischen Ländern und Frankreich (in Prozent des BIP)}

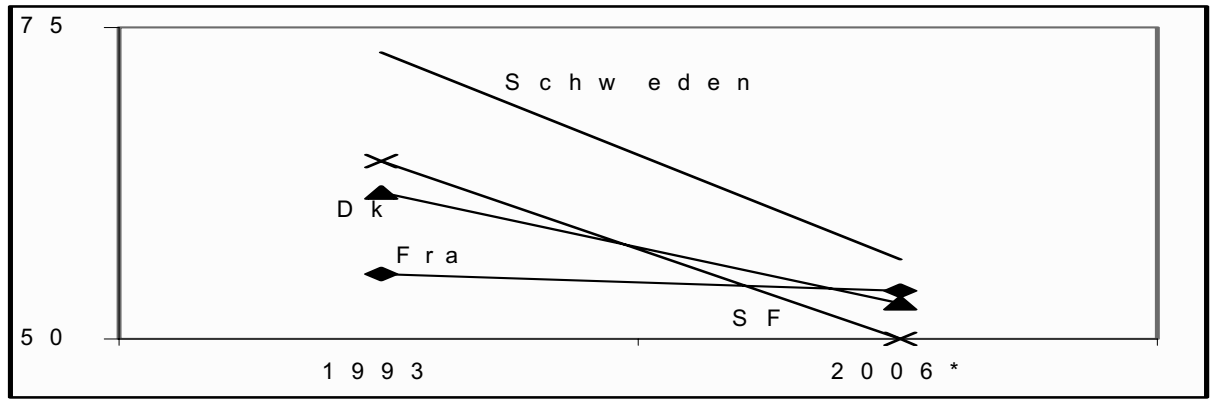

* Schätzung

Quelle: European Commission, DG EcFin, European Economy, No.6/2004, S. 560

Ferner fällt auf, dass die nordischen Mitglieder der Europäischen Union im Hinblick auf die Einnahmenseite ihrer Haushalte durchaus nicht homogen sind - weder untereinander, noch im Vergleich zu anderen Industrieländern (Tab. 1). Dänemark weist die stärkste Lohnsteuerbelastung der Arbeitseinkommen auf und hält im Gegenzug als Standortvorteil die Lohnnebenkosten gering. Finnland hat unter den nordischen Ländern die geringste Staatsund Steuerquote; der finnische Fiskus vertraut aber stärker auf die Körperschaftssteuer als 
Einnahmenquelle. Sie hat bisher - bei in etwa gleichen nominalen Steuersätzen - einen gröBeren Anteil am Steueraufkommen Helsinkis als in Schweden und einen deutlich größeren als in Dänemark gehabt (Tab. 1, vorletzte Zeile). Damit könnte aber jetzt Schluss sein: 2005 hat sich Finnland dem Steuerwettbewerb in der EU angeschlossen und die nominalen Körperschaftssteuersätze um drei Prozentpunkte auf 26 Prozent gesenkt. Steuerpolitisches Fundament bleibt die bereits vor 15 Jahren eingeleitete Umschichtung der Steuerbelastung vom mobilen Faktor Kapital auf den weniger mobilen Faktor Arbeit.

Im weiteren internationalen Vergleich melden die nordischen Länder und zahlreiche kontinentaleuropäische Ökonomien zwar ähnliche Steuerquoten. Sie können jedoch zum Teil sehr unterschiedlich strukturiert sein. Beispielsweise ist der Anteil der Sozialversicherungsbeiträge und damit der Lohnnebenkosten in Frankreich und in Deutschland größer als in Finnland (und erst recht als in Dänemark). Dennoch erzielt das skandinavische Land bei gleicher Arbeitslosenrate wie in Frankreich höhere Wachstumsraten und relativ größere Steuereinnahmen. Neben Dänemark und Finnland schafft es auch Schweden, die Lohnnebenkosten im Rahmen zu halten und fördert somit das Wachstum. Im Resultat erzielen alle drei Skandinavier Budgetüberschüsse, während Frankreich und Deutschland regelmäßig den Stabilitäts- und Wachstumspakt verletzten.

Alles in allem sind die Unterschiede im Staats- und Steuermodell der Skandinavier gröBer als die Gemeinsamkeiten. Trotzdem sind ihre Finanzen besser als in anderen Ländern darauf orientiert, das Wachstum nicht abzubremsen und positive Haushaltssalden zu erzielen. Im Unterschied zu den langsam wachsenden Mitgliedsländern ist die fiskalische Haupteinnahmenquelle die Besteuerung der persönlichen Einkommen; die Besteuerung der Gewinne sowie die Belastung der Arbeit mit Sozialversicherungsbeiträgen ist dagegen von geringerer Bedeutung. In dieser Hinsicht ähnelt die skandinavische Steuerstruktur jener der angelsächsischen Länder beziehungsweise der USA, die im Lissabon-Prozess als Benchmark für Wachstum und Innovation fungieren (Tab 1).

Tabelle 1: Steuerindikatoren nach Ländern 2005 (in Prozent des BIP)

\begin{tabular}{|l|l|l|l|l|l|l|l|}
\hline & Dänemark & Finnland & Schweden & $\begin{array}{c}\text { Deutsch- } \\
\text { land }\end{array}$ & Frankreich & USA* \\
\hline Staatsquote & 53,7 & 50,6 & 56,6 & 46,6 & 53,9 & 33,8 \\
\hline Steuerquote & 48,6 & 44,4 & 50,2 & 39,5 & 44,8 & 25,7 \\
\hline Anteil an den Gesamtsteuereinnahmen ( in Prozent, 2000, ohne, ,sonstige Einnahmen“) \\
\hline indirekte Steuern & 30,8 & 28,5 & 20,0 & 27,2 & 25,2 & 20,0 \\
\hline Einkommenssteuer & 52,7 & 30,7 & 35,6 & 25,3 & 18,1 & 35,0 \\
\hline Körperschaftssteuer & 4,9 & 11,7 & 7,6 & 4,7 & 7,1 & 10,0 \\
\hline SV-Beiträge & 4,5 & 25,6 & 28,0 & 39,1 & 36,2 & 27,0 \\
\hline
\end{tabular}

*teilweise geschätzt

Quelle: EU-Kommission, DG EcFin, European Economy, No. 6/2004, Statistical Annex; OECD, Taxing Wages, http://www.oecd.org/dataoecd/26/8/2495227.pdf; UN ECE, Economic Survey of Europe, 1/2004, Table 5.3.3, 5.3.5

\section{Lehren aus Skandinavien: Qualität geht vor Quantität}

Das nordische Wirtschafts- und Sozialmodell zeichnet sich durch eine aktive Rolle des Staates und ein hohes Maß an gesellschaftlicher Kohäsion aus. Der Gini-Koeffizient als $\mathrm{Ma} ß$ der Armutsrate ist in Skandinavien OECD-weit am geringsten, da die Einkommensun- 
gleichheit über Besteuerung und Transfers spürbar abgebaut wird. Die skandinavische Konsensgesellschaft ermöglicht eine überdurchschnittliche Staatsquote, da das Vertrauen in die positive Fürsorgerolle des Staates groß ist.

Der hohe Bildungs- und Qualifizierungstand ermöglicht hohe Produktivitäts- und Einkommensniveaus in allen drei Ländern und gibt ihnen genügend Spielraum, sich den wechselnden Anforderungen der Märkte anzupassen. Der vergleichsweise kleine Markt, gepaart mit großen Exportquoten, macht Finnland und Dänemark flexibel in der internationalen Arbeitsteilung. Eine rückläufige Exportnachfrage aus den langsam wachsenden Mitgliedsländern können sie durch ihre Umorientierung auf andere Partner schneller als die großen Volkswirtschaften kompensieren.

Für die BIP-Wachstumsrate sind aber nicht allein Flexibilität und günstige weltwirtschaftliche Bedingungen ausschlaggebend. Insbesondere in Hochlohnwirtschaften ist das Verbreitungstempo des technologischen Fortschritts, beziehungsweise die Rate der totalen Faktorproduktivität (TFP), wichtig, weil so das Produktivitäts- und Einkommenswachstum beschleunigt wird. Die Analyse zeigt, dass gemessen an der im Lissabon-Prozess der EU als Benchmark dienenden US-Wirtschaft das Tempo des technischen Fortschritts in den nordischen Mitgliedern der Europäischen Union seit 1996 zugelegt hat. In anderen Industrieländern, darunter Frankreich, Deutschland und die Eurozone, ging es dagegen zurück, mit der unmittelbaren Folge eines langsamen BIP-Wachstums (Abb. 4).

\section{Abbildung 4: Jahresdurchschnittliche Wachstumsrate der technologischen Fort- schritts (TFP) in der EU und den USA (Benchmark 1996-2005), in Pro- zent}

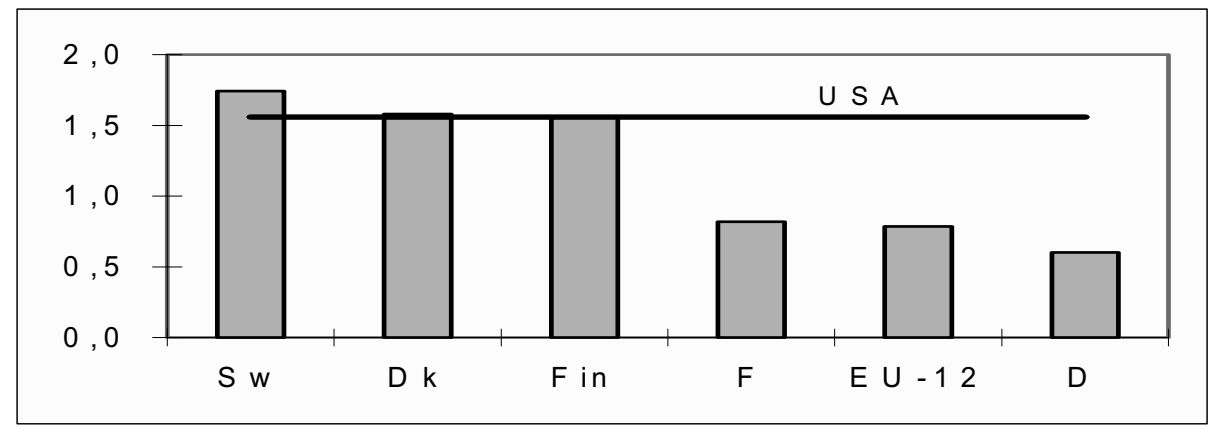

Quelle: European Commission, DG EcFin, European Economy, No. 6/2004, Statistical Annex

Während Dänemarks, Finnlands und Schwedens Wachstum stark technologie- und wenig arbeits- beziehungsweise kapitalbasiert ist, hat sich dagegen in Deutschland das kapitalbasierte Wachstumsmodell verfestigt. Das ist wenig erfreulich, da sich die deutsche Wirtschaft relativ nah am Steady State befindet - einem Zustand bei dem weitere Anstrengungen zum Ausbau des Kapitalstocks mit immer weniger Wachstum quittiert werden. Ähnliches gilt für Frankreich und für die Eurozone als Ganzes, für die ähnlich wie für Deutschland eine abnehmende BIP-Wachstumsrate sowie die inzwischen negative Kapitalproduktivität symptomatisch sind.

Angesichts solcher Trends ist die Zielsetzung der Skandinavier, gemäß der LissabonStrategie ein dynamisches wissensbasiertes Wachstum zu erzielen, nicht nur richtig, sondern auch von enormer Bedeutung für ihre zukünftige ökonomische Entwicklung. In dieser Hin- 
sicht können sie ungeachtet ihres vergleichsweise kleinen Marktes den großen kontinentalen europäischen Ländern mit Wachstumsproblemen Vorbild sein. So würden langsam wachsende Ökonomien bei einem vermehrten Einsatz neuer Produktions- und Organisationsverfahren, einschließlich der Steigerung der Qualifikation der Arbeit, spürbare Wachstumseffekte erzielen. Wegen des schleppenden TFP-Wachstums in der EU müsste das LissabonZiel, 3 Prozent des gemeinschaftlichen BIP für Investitionen in Innovation, Technologie und Bildung (F\&E) auszugeben, sogar nach oben korrigiert werden. Mittelfristig wird selbst bei einer Aufstockung der Ausgaben für Forschung und Entwicklung (F\&E) von derzeit 1,9 Prozent auf 3 Prozent (jetziges Lissabon-Ziel) der Wachstumseffekt gering bleiben. Ursache ist das langsame TFP-Wachstum in den großen kontinentaleuroropäischen Länder, so dass mehr - selbst um ein Drittel - F\&E-Mittel das BIP-Wachstum mäßig beschleunigen würde. In den skandinavischen Mitgliedstaaten der Europäischen Union sind die Resultate günstiger - ein Hinweis darauf, dass das Lissabon-Ziel eines wissensbasierten Wachstums in der EU nicht unerreichbar ist. ${ }^{13}$

Ein Zusammenhang zwischen Technologiewachstum und Beschäftigung besteht ebenfalls: Ein durch einen kräftigen Technologieschub initiiertes BIP-Wachstum würde als erstes die konjunkturell bedingte Arbeitslosigkeit abbauen, da ab einer Wachstumsrate der Wirtschaft von 2 Prozent pro Jahr die Freisetzung von Arbeit aufhört. Die derzeit sehr hohe Arbeitslosenrate in einigen Mitgliedsländern würde dadurch sukzessive verringert werden. In Finnland ist dieser Weg erfolgreich gegangen worden, mit dem Ergebnis, dass die sehr hohe Arbeitslosigkeit der 1990er Jahre durch das schnelle Wachstum abgebaut werden konnte. Es zeigt sich, dass das Tempo des technologischen Fortschritts für die BIP-Wachstumsrate entscheidend ist, da der Beitrag der TFP zum Wachstum sehr groß ist - zwischen einem Drittel und drei Vierteln (Tabelle 2). Innovation, Modernisierung und der Strukturwandel zugunsten der Industrien mit hohem Informations- und Kommunikationsanteil wirken wachstumsfördernd; dagegen wirkt jedwede Konservierung alter Strukturen wachstumsbremsend.

Tabelle 2: Tempo und Beitrag des technischen Fortschritts (TFP) zum Pro-KopfWirtschaftswachstum in der EU (Durchschnittswerte 1996-2005)

\begin{tabular}{|l|c|c|c|}
\hline \multirow{2}{*}{ Land } & \multicolumn{2}{c|}{$\begin{array}{c}\text { Wachstumsrate } \\
\text { (in Prozent p.a.) }\end{array}$} & $\begin{array}{c}\text { TFP-Beitrag zum } \\
\text { Wachstum } \\
\text { (in Prozent) }\end{array}$ \\
\hline Schweden & BIP pro Kopf & TFP & 73 \\
\hline Dänemark & 2,4 & 1,7 & 72 \\
\hline Finnland & 2,2 & 1,6 & 62 \\
\hline Deutschland & 2,5 & 1,6 & 50 \\
\hline Frankreich & 1,2 & 0,6 & 46 \\
\hline EU-12 & 1,8 & 0,8 & 37 \\
\hline
\end{tabular}

Berechnet nach: European Commission, DG EcFin, European Economy, No. 2/2004, Statistical Annex.

13 Im Zeitraum 1996 bis 2005 lag das BIP-Wachstum in der Eurozone bei durchschnittlich 1,6 Prozent p.a. mit einer TFP-Rate von unter 0,5 Prozent p.a. In den nordischen Mitgliedern der Europäischen Union betrugen die entsprechenden Indikatoren im selben Zeitraum 2,5 und 1,8 Prozent p.a. Quelle: Ognian N. Hishow: Wachstumspolitik in der EU. Wirtschaftsleistung, Beschäftigung und Innovation vor dem Hintergrund der LissabonAgenda. Studie S23, Stiftung Wissenschaft und Politik, Berlin 2005, Tabelle A1. 
Insbesondere im Falle Schwedens und Dänemarks liegt dies klar auf der Hand: In beiden Ländern ist der Kapitalstock relativ zum BIP inzwischen groß (jeweils 3,3 und 3 in den späten 1990er Jahren). Versuche, ihn weiter auszubauen, würden auf Grund sinkender Wachstumswirksamkeit der Investitionen erfolglos bleiben. Auch ist die Möglichkeit, durch Erschließung freier Arbeitsreserven das Wachstum zu beschleunigen, in beiden Ländern mit ihren hohen Beschäftigungsquoten begrenzt. ${ }^{14}$ Daher war es nur konsequent, auf eine schnelle Implementierung neuer Technik und Arbeitsorganisation als Wachstumsinput zu setzen. Die Kombination aus höherem Tempo des technischen Fortschritts und seinem gröBeren Anteil an der BIP-Wachstumsrate im Durchschnitt der Jahre 1996-2005 in den nordischen Ländern als in Deutschland (Tab. 2) erklärt hinreichend ihre sehr viel bessere aktuelle ökonomische Verfassung, insbesondere vor dem Hintergrund der Herausforderungen durch Osterweiterung und Globalisierung.

\section{Ist das nordische Wirtschafts- und Sozialmodell kopierfähig?}

Bei der Diskussion um ein geeignetes europäisches Wirtschafts- und Sozialmodell, das Wohlstand nachhaltig sichert und die Herausforderungen der Zukunft erfolgreich bewältigt, wird wiederholt auf die Verträglichkeit eines generösen Sozialstaates mit guten wirtschaftlichen Erfolgen in den drei nordischen Mitgliedern der Europäischen Union hingewiesen. Alle drei Länder erzielen trotz - oder wegen - ihrer großen Staatsquote zukunftssichernde Haushaltsüberschüsse und melden vergleichsweise geringe Einkommensungleichgewichte.

Die direkte Übertragung der nordischen Erfahrungen auf die großen europäischen ,Problemwirtschaften' wie Deutschland oder Frankreich ist bei aller formalen Attraktivität des Modells freilich wenig hilfreich. Die hohe Steuerquote bewirkt, dass der Privatverbrauch in den skandinavischen Ländern langsamer steigt als im OECD-Durchschnitt. Während das für die kleinen, sehr offenen Volkswirtschaften Dänemarks, Finnlands und Schwedens weniger problematisch ist, ist der Privatverbrauch in den großen Ländern die wichtigste Nachfragekomponente. ,Mehr Staat' würde hier das Wachstum konterkarieren, nicht fördern. Das haben auch die Skandinavier erkannt und ihre Staatsquote spürbar gekürzt.

Zugleich - und das ist eine der bemerkenswerten Leistungen - haben die Skandinavier ein Abrutschen in die Staatsverschuldung vermeiden können. Mit der Einführung einer ,Dualen Einkommensteuer" seit den späten 80ern und den frühen 90ern wurde der Unternehmenssektor entlastet. Dual steht für die unterschiedliche Steuerbelastung von Einkommen aus Arbeit und Vermögen bzw. Kapitalbesitz: Während Arbeitseinkommen progressiv besteuert werden, unterliegen Gewinne, Zinseinnahmen und Dividenden einer Flat Tax zu international moderaten Sätzen, die weit unter den deutschen Nominalsätzen liegen. Die Steuersatzsenkungen ab den frühen 1990er Jahren haben dazu beigetragen, dass diese Länder im Vergleich zu Deutschland weniger unter Gewinnverlagerungen leiden. Fördernd für die Standortattraktivität ist die Tatsache, dass die Haupteinnahmenquelle des Fiskus die persönliche Einkommenssteuer ist, gefolgt von den Umsatz- und Verbrauchssteuern. Die Belastung mit Lohnnebenkosten ist speziell in Dänemark im internationalen Vergleich minimal.

Der Erfolg der nordischen Mitglieder der Europäischen Union ist eine Kombination aus Gegebenheiten (kleine offene Ökonomien, gewachsenes Vertrauen in die Fürsorgefunktion des Staates, Naturressourcen in Schweden und Finnland) und genuinen Leistungen (hoher

14 Formal gesehen liegen solche Reserven insbesondere in Schweden vor: Hier ist die Krankenquote im internationalen Vergleich besonders hoch, was ein Indiz dafür ist, dass Arbeitsinput ,verschenkt " wird. ,Krankfeiern“ ist ein Substitut für bezahlte Freizeit, die auf diese Weise ausgedehnt wird; somit ist dieses Problem kurzfristig nicht zu lösen. 
Bildungsstand und damit hohe Produktivität und Arbeitseinkommen, Anpassungsfähigkeit im internationalen Wettbewerb, effiziente Verwaltung und Steuerung staatlicher Zuwendungen). Es ist nicht der, große Staat " per se, der Skandinavien den Erfolg beschert; folglich ist von jedwedem mechanischen Kopieren des nordischen Wirtschafts- und Sozialmodels durch andere Nationen mit anderen Voraussetzungen abzuraten.

Auch sind einige Sonderfaktoren nicht zu unterschätzen: In Schweden fördert die unabhängige nationale Geld- und Wechselkurspolitik den Aufschwung. Die schwedische Krone wurde in der Rezession gegenüber dem Euro und dem britischen Pfund real abgewertet, um die schwedischen Exporte zu beleben.

Hilfreich ist auch die skandinavische Neigung, Konflikte im Konsens zu lösen, die in allen drei Ländern für moderate Lohnabschlüsse im Sinne von Wachstum und Beschäftigung sorgt.

Nicht zuletzt spielt der Sonderfaktor Naturkapital eine positive Rolle, da er in Schweden und Finnland bestimmten Industrien (Holz, Papier) komparative Vorteile gegenüber der internationalen Konkurrenz verschafft und so selbst in wirtschaftlichen Schwächephasen die Gesamtnachfrage stützt.

Verschont von Problemen sind die Länder freilich nicht: Insbesondere in Finnland verharrt die Arbeitslosigkeit auf einem hohen Stand und liegt damit um bis zu vier Prozentpunkte über der Leistung der angelsächsischen Volkswirtschaften. Zusammen mit Schweden erlebte das Land Anfang der 1990er Jahre die schwerste Rezession unter der westlichen Industrieländern; in beiden Volkswirtschaften ging die Wirtschaftsleistung präzedenzlos drei Jahre hintereinander zurück.

Dieser makroökonomische Schock entfaltete jedoch eine heilsame Wirkung: Finnland restrukturierte zum Preis massiver Entlassungen seine Wirtschaft und betrat anschließend wieder einen Wachstumspfad. Schweden kürzte radikal - von 73 Prozent 1993 auf gegenwärtig 56,5 Prozent des BIP - seine Staatsquote und glich sie damit dem Rest der meisten Mitgliedsländer an. Im öffentlichen Sektor wurden Entlassungen durchgesetzt und die Zumutbarkeitskriterien für die Arbeitsaufnahme bei Arbeitslosigkeit wurden gestrafft.

Diese ,Peitsche“ ist jedoch nur die eine Seite der Arbeitsmarktpolitik: Als Zuckerbrot wurde die aktive Beschäftigungspolitik ausgebaut. Unter Berücksichtigung der Teilnehmer von Beschäftigungsprogrammen wäre die Arbeitslosenrate effektiv um bis zu 2 Prozentpunkte höher. Diese Entschlossenheit, die Arbeitsaufnahme von Arbeitslosen aktiv zu fördern und zu fordern, ist nachzuahmen.

Unbedingt übernahmewürdig ist die Innovationsfreudigkeit der nordischen Volkswirtschaften, die ihnen Wettbewerbs- und damit Wachstums- und Haushaltsvorteile verschafft. Auch die ideologiefreie Kreativität bei der Standortsicherung ist in den Ländern kopierfähig: Dänemark war stets bemüht, seinen Wirtschaftsstandort durch eine unternehmensfreundliche Steuerpolitik und durch geringe Lohnnebenkosten attraktiv zu halten. Selbst im Vergleich zu wirtschaftliberalen Ländern wie den USA, Großbritannien oder Irland ist die Steuerbelastung der Unternehmen in Dänemark geringer. Das sichert den Standort Dänemark, der im internationalen Vergleich ansonsten kein ausgeprägter High-Tech-Standort ist.

Ein einheitliches nordisches Beschäftigungs- und Steuermodell existiert folglich nur bedingt. Jedoch gibt es zahlreiche sozial- und wirtschaftspolitische Elemente, die für andere EU-Staaten als Orientierung dienen können. 